\title{
Perfil e expectativas profissionais de concluintes do curso de graduação em Odontologia da Universidade Federal de Santa Catarina
}

\author{
Carolina Vieira da Silva*; Vinícius Spiger**; Cláudio José Amante*** \\ * Cirurgiã-dentista, Pesquisadora, Universidade Federal de \\ Santa Catarina \\ ** Cirurgião-Dentista, mestrando no Programa de Pós- \\ Graduação em Odontologia da Universidade Federal de \\ Santa Catarina \\ *** Doutor em Engenharia de Produção, Professor do \\ Departamento de Odontologia, Universidade Federal de \\ Santa Catarina
}

Recebido em 28/09/2017. Aprovado em 20/06/2018.

\begin{abstract}
RESUMO
A Odontologia vivencia constantes mudanças, em uma realidade de saturação do mercado, fortalecimento do setor público e novos desafios profissionais. A formação universitária almeja um novo perfil acadêmico, conforme as Diretrizes Curriculares Nacionais. Este estudo objetivou verificar o perfil dos estudantes do último ano do Curso de Graduação em Odontologia da Universidade Federal de Santa Catarina e suas expectativas profissionais. Um estudo descritivo transversal foi conduzido por meio de um questionário autoaplicável, composto por questões sociodemográficas e sobre a expectativa profissional dos estudantes. De 84 estudantes, 90,48\% concordaram em participar do estudo. A amostra foi majoritariamente composta por estudantes do sexo feminino $(69,74 \%)$, brancos $(92,10 \%)$, solteiros $(88,16 \%)$ e com idade média de 24 anos. Quanto à formação, 64,47\% cursaram o ensino médio em escola privada. Renda familiar média de 5 a 10 salários mínimos foi declarada por $39,47 \%$ dos participantes e $84,21 \%$ afirmaram não trabalhar. Apenas $18,42 \%$ dos estudantes ingressaram no curso por meio de ações afirmativas. Em relação às expectativas profissionais, $80,26 \%$ querem trabalhar no serviço público, principalmente por estabilidade $(42,10 \%)$ e renda complementar ao serviço privado $(40,79 \%)$. A principal dificuldade esperada pelos alunos é a saturação do mercado de trabalho $(69,74 \%)$. Todos almejam cursar algum tipo de pós-graduação, principalmente nas áreas de Prótese Dentária (36,84\%), Dentística (34,21\%) e Implantodontia $(32,90 \%)$. Neste estudo, identificou-se uma tendência às especialidades odontológicas no perfil dos estudantes, embora o emprego público seja almejado pela maioria, além de constante preocupação com a saturação do mercado de trabalho.
\end{abstract}

Descritores: Educação em Odontologia. Mercado de Trabalho. Recursos Humanos em Odontologia. 


\section{INTRODUÇÃO}

Concebida devido às necessidades humanas, a Odontologia vivenciou diversas transformações em seu processo de trabalho, incorporando crescente complexidade, que convergiu para significativos impactos e mudanças no perfil profissional do cirurgiãodentista ${ }^{1}$.

Por muito tempo, o modelo de trabalho da Odontologia foi direcionado à prática individual, tecnicista e curativa ${ }^{1}$, majoritariamente centrado no mercado assistencialista e privado. Entretanto, após a década de oitenta, crescente foi a tendência dos profissionais ao assalariamento, ao trabalho com convênios e ao credenciamento ${ }^{2}$.

No contexto nacional, observa-se uma complexa situação referente ao mercado de trabalho. O país concentra cerca de $20 \%$ dos cirurgiões-dentistas de todo o mundo, com marcante má-distribuição territorial, o que implica tanto em desigualdades no mercado de trabalho quanto em dificuldades no acesso aos serviços odontológicos ${ }^{3}$. Conforme dados publicados em março de 2016 pelo Conselho Federal de Odontologia, são 278.394 os cirurgiões-dentistas inscritos na entidade ${ }^{4}$.

Entretanto, o marco principal no Brasil se relaciona à consolidação do Sistema Único de Saúde (SUS). Fruto de movimentos relacionados à Reforma Sanitária, o SUS firmou-se pela Constituição Federal de 1988, emergindo como o modelo de saúde vigente no país, de caráter público e orientado para um modelo de promoção e de proteção da saúde ${ }^{1}$.

Também a criação do Programa Saúde da Família, em 1994, e a posterior inclusão da Saúde Bucal na Estratégia Saúde da Família, no ano de 2000, corroboraram para uma nova lógica na atuação profissional do cirurgião-dentista ${ }^{5}$. Pode-se evidenciar tais mudanças pela observação do número de cirurgiões-dentistas inseridos nos serviços públicos de saúde, estimado em cerca de 122.125 profissionais, conforme dados do DataSUS ${ }^{6}$.

Desta maneira, a expansão da atuação pública, as mudanças no perfil populacional e a própria reorientação da Odontologia implicaram em uma significativa mudança na atuação do cirurgião-dentista, seja ela vinculada ao poder público, credenciada, assalariada ou liberal ${ }^{1,3}$.

Concomitante a estas mudanças, também o cenário educacional sofreu importantes alterações, como a inclusão de áreas como a Psicologia, a Antropologia, a Sociologia e a metodologia científica ainda na década de oitenta, por meio da Resolução $\mathrm{n}^{\circ} 4$, datada de 3 de setembro de 1982, indicando a ampliação do escopo na formação profissional ${ }^{7}$.

Apesar desta mudança inicial, uma completa reordenação da formação em Odontologia era necessária para adequar-se à realidade brasileira e às suas necessidades. Com a publicação das Diretrizes Curriculares Nacionais (DCN), no ano de 2002, efetuou-se mudança de foco de um caráter estrito para um perfil mais generalista, contemplando várias habilidades que transcendiam a pura prática clínica.

Assim, as DCN foram responsáveis por um processo de reformas curriculares nos cursos brasileiros de graduação em Odontologia, objetivando o equilíbrio entre a excelência técnica e a relevância social, transferindo o foco da educação para o estudante, em prol de um perfil capacitado para a assistência humanizada, de alta qualidade e resolutiva, incluindo a priorização dos aspectos relativos à promoção da saúde ${ }^{8,9}$.

Ainda assim, as análises do ensino superior odontológico apontam para a insuficiência 
formativa, quando considerado o impacto nos programas públicos e coletivos na área da saúde bucal $^{10}$. Estudantes refletem uma visão da prática profissional como especializada e orientada majoritariamente por interesses mercadológicos ${ }^{11}$.

No mesmo caminho seguiu a Universidade Federal de Santa Catarina (UFSC) e o Curso de Graduação em Odontologia passou por reformas que objetivaram o melhor preparo possível para seus estudantes, adequando-se ao novo perfil exigido para o profissional. O novo Projeto Político Pedagógico que reorientou o curso foi implementado em 2005, e o início do processo de reforma curricular se deu no primeiro semestre de $2007^{12}$.

Dentro do contexto institucional da UFSC, um estudo conduzido em 2013 objetivou estabelecer o perfil socioeconômico dos estudantes. Observou-se a predominância de indivíduos do sexo feminino, com idade entre 18 e 24 anos, brancos, solteiros, sem filhos e pertencentes à classe $\mathrm{B}^{13}$.

Considerando as mudanças do perfil profissional do cirurgião-dentista e do atual mercado de trabalho, bem como as necessárias adequações da formação em Odontologia, este estudo teve como objetivo descrever o perfil dos estudantes do último ano do Curso de Graduação em Odontologia da UFSC e suas expectativas em relação à vida profissional.

\section{METODOLOGIA}

O presente estudo foi realizado após sua plena aprovação pelo Comitê de Ética em Pesquisa com Seres Humanos da Universidade do Estado de Santa Catarina (UDESC), em abril de 2015, sob o CAEE 43625815.6.0000.0121 e sob o parecer de $n^{\circ} 1.037 .414$.

Foi conduzida uma pesquisa descritiva, do tipo transversal, aplicada a alunos regularmente matriculados no $9^{\circ}$ e $10^{\circ}$ semestres do Curso de Graduação em Odontologia da UFSC.

Os dados foram coletados em sala de aula, após a autorização do professor responsável, seguida da apresentação dos objetivos da pesquisa, de seus riscos e benefícios, bem como do questionário de coleta de dados. As dúvidas do estudo foram clarificadas e o Termo de Consentimento Livre e Esclarecido entregue aos voluntários.

Os alunos que concordaram em participar do estudo receberam o instrumento de coleta de dados, desenvolvido por Machado et al., ${ }^{14} \mathrm{e}$ adaptado pelos autores, composto por questões objetivas abordando informações sobre o perfil socioeconômico e expectativas quanto à carreira profissional.

Os dados obtidos foram analisados por intermédio de medidas estatísticas descritivas.

\section{RESULTADOS}

No momento da coleta de dados, 84 acadêmicos estavam matriculados no último ano do Curso de Graduação em Odontologia da UFSC. Destes, 76 aceitaram participar deste estudo, representando uma taxa de resposta de 90,48\%. A análise do perfil dos estudantes do último ano do curso de Odontologia demonstrou predominância do sexo feminino, com 53 alunas $(69,74 \%)$, em relação aos $23(30,26 \%)$ estudantes do sexo masculino. A idade de vinte quatro anos foi a mais observada $(28,95 \%)$, com apenas dois alunos $(2,63 \%)$ com mais de trinta anos. A população foi majoritariamente composta por indivíduos autodeclarados brancos $(92,10 \%)$, solteiros $(88,16 \%)$ e sem filhos $(97,37 \%)$.

Quanto à formação, 64,47\% dos entrevistados afirmaram ter cursado todo o ensino médio em escola privada. Além disso, $55,26 \%$ das mães e $60,52 \%$ dos pais apresentaram também ensino superior completo. Em relação à renda familiar, 39,47\% dos alunos declararam estar na faixa de 5 a 10 salários mínimos e $17,11 \%$ superior a 15 salários mínimos. Além disso, 84,21\% dos estudantes relataram não trabalhar, tendo todos os seus 
gastos financiados pela família, e 14,47\% apontaram trabalhar e receber auxílio da família. Entre todos os participantes, somente um $(1,32 \%)$ relatou não receber ajuda financeira familiar.

Quanto ao ingresso do acadêmico na instituição, 18,42\% afirmaram terem ingressado por políticas de ação afirmativa, sendo que $10,53 \%$ ingressaram pelo sistema de reserva de vagas com recorte social (escola pública, vulnerabilidade social) e $6,56 \%$ pelo sistema de reserva com identificação étnico-racial (negros, pardos e indígenas).

No aspecto habitacional, $35,53 \%$ dos estudantes relataram morar com os pais, enquanto $27,63 \%$ informaram morar com amigos, $25,00 \%$ sozinho e apenas $11,84 \%$ com cônjuge e/ou filhos. Todos os participantes relataram possuir computador e acesso à internet em sua residência.

Além do perfil dos estudantes do último ano do curso, este estudo também avaliou suas expectativas profissionais. Entre os participantes, $64,47 \%$ relataram que pretendem associar o trabalho em consultório particular e serviço público, enquanto $19,74 \%$ almejam trabalhar de maneira exclusiva em consultório privado. Somente $15,79 \%$ dos alunos esperam ter dedicação exclusiva ao serviço público.

Quando questionados quanto aos motivos para almejar o trabalho no serviço público, $42,10 \%$ dos participantes relataram a estabilidade profissional. O segundo motivo mais relatado foi a possibilidade de renda acessória ao trabalho na iniciativa privada $(40,79 \%)$. Além disso, 26,32\% dos alunos afirmaram que o serviço público é uma boa alternativa como primeiro emprego. O lucro foi apontado como razão por apenas $6,58 \%$ dos participantes.

Outra questão avaliada por este estudo foi a expectativa em relação à renda salarial. Entre os participantes, $47,37 \%$ esperam uma renda entre 4-6 mil reais ao mês, enquanto $34,21 \%$ demonstraram expectativa de mais de 6 mil reais mensais. Apenas $1(1,31 \%)$ tem expectativa de receber entre mil e 2 mil reais ao mês, 6,58\% relataram expectativa entre 2 e 3 mil reais e $10,53 \%$ entre 3 e 4 mil reais mensais.

Entre as dificuldades que os estudantes esperam encontrar no exercício da profissão estão a saturação no mercado de trabalho $(69,74 \%)$, a insuficiente condição financeira da população $(32,90 \%)$, a falta de preparo profissional $(18,42 \%)$ e a falta de informação da comunidade $(11,84 \%)$. Somente $7,89 \%$ dos estudantes afirmaram não ter expectativa quanto a problemas na vida profissional.

Dentre os estudantes, $10,53 \%$ informaram o desejo de cursar outra graduação e todos afirmaram ter interesse em prosseguir seus estudos após a graduação. A Tabela 1 apresenta as áreas de concentração de maior interesse entre os participantes.

Em relação ao cadastro no Conselho Federal de Odontologia, 23,70\% dos estudantes relataram não ter pensado no assunto e 3,95\% afirmaram que não desejam realizar o cadastro no conselho.

\section{DISCUSSÃO}

Embora historicamente a Odontologia seja considerada uma profissão tipicamente masculina, observa-se a feminização da profissão no mercado de trabalho brasileiro, onde as mulheres representam cerca de $51,2 \%$ dos dentistas ${ }^{1,3,14,15}$. A presença feminina no meio acadêmico odontológico também foi observada no presente estudo.

O perfil do cirurgião-dentista brasileiro é principalmente o do profissional jovem, geralmente em uma faixa etária entre os 26 e 35 anos, demonstrando o quão cedo estes adentram o mercado de trabalho ${ }^{3}$. Os estudantes do último ano da UFSC, em sua maioria na média de 24 anos de idade, vivenciam essa situação, observada também em outras universidades ${ }^{16}$. 
Em estudo realizado com estudantes do primeiro semestre da Faculdade de Odontologia da Universidade de São Paulo, observou-se uma média de idade de 19,5 anos, indicando que os estudantes iniciam o curso ainda jovens ${ }^{15}$. Na Universidade Federal do Rio Grande do Norte, observou-se que a maior parte dos egressos se insere rapidamente no mercado de trabalho ${ }^{17}$.

Tabela 1. Distribuição das áreas de interesse da Pós-Graduação em Odontologia, em frequências absoluta (n) e relativa (\%), entre os estudantes do último ano do Curso de Graduação em Odontologia da UFSC

\begin{tabular}{|c|c|c|}
\hline Área de Concentração & $\mathbf{n}$ & $\%$ \\
\hline Prótese Dentária & 28 & 36,84 \\
\hline Dentística & 26 & 34,21 \\
\hline Implantodontia & 25 & 32,90 \\
\hline Endodontia & 23 & 30,26 \\
\hline Ortodontia e Ortopedia Facial & 18 & 23,68 \\
\hline Cirurgia e Traumatologia Bucomaxilofacial & 14 & 18,42 \\
\hline Odontopediatria & 11 & 14,47 \\
\hline Peridontia & 9 & 11,84 \\
\hline Saúde Coletiva & 8 & 10,53 \\
\hline Radiologia & 3 & 3,94 \\
\hline Total & 76 & 100,00 \\
\hline
\end{tabular}

De forma geral, o perfil socioeconômico dos estudantes do último ano apresentou-se favorável, tendo a maior parte das famílias uma renda mensal elevada, serem sustentados pela família, terem seus gastos supridos por terceiros, tendo estudado em colégios privados, com bom entendimento de um segundo idioma, escolaridade materna e paterna elevadas e acesso a computadores e à internet. Essas condições corroboram com o observado em outras universidades, onde o nível socioeconômico elevado é observado entre os estudantes de Odontologia ${ }^{10,11,16}$.

Tais características no perfil dos estudantes proporcionam como vantagem a possibilidade de maior conforto para desenvolver as atividades durante o curso de graduação com dedicação exclusiva $^{14}$. Por outro lado, há um crescente debate no sentido de ampliar o acesso ao curso às classes com indicadores socioeconômicos menos favoráveis. Nesse sentido, a UFSC possui um processo seletivo com reserva de vagas para ações afirmativas desde 2008, embora nem um terço dos participantes tenha relatado ter sido beneficiado. Em 2016, a UFSC passou a reservar $50 \%$ de suas vagas para ações afirmativas, o que pode implicar em uma mudança do perfil socioeconômico dos alunos, exigindo maior atenção por parte do curso frente às novas possibilidades de perfil entre os alunos, em contrapartida à polarização atualmente observada.

Em relação ao exercício da profissão, observa-se que os anseios demonstrados pelos alunos é um reflexo da nova situação da Odontologia, onde a progressiva incorporação de tecnologias, da especialização, a redução do exercício liberal, o aumento do percentual de 
profissionais com vínculo público e a incorporação da Saúde Bucal no Programa de Saúde da Família reforçam as novas realidades para atuação profissional ${ }^{3}$.

A maioria dos alunos relatou que deseja trabalhar no serviço público, fato que pode ser observado em diferentes pesquisas sobre a perspectiva de estudantes de Odontologia. Há consciência das dificuldades profissionais e da segurança financeira derivada do emprego público.

Dos alunos participantes da pesquisa, $79 \%$ desejam trabalhar no serviço público, sendo de forma exclusiva ou não. Esse desejo não é exclusivo dos alunos da UFSC, diversas pesquisas sobre as perspectivas do graduando quanto ao seu futuro profissional chegaram ao mesmo resultado, os dados obtidos junto aos estudantes sugerem que há consciência das dificuldades profissionais e que um emprego proporciona maior segurança financeira ${ }^{2,16}$. Em um estudo realizado com estudantes de graduação em Odontologia na Universidade Federal da Paraíba, 76,7\% dos alunos relataram ter interesse em associar o serviço público com o serviço privado, e $16,8 \%$ ter interesse exclusivo no serviço público ${ }^{14}$. Na pesquisa de Pinheiro et al., ${ }^{17}$, observou-se que apenas $9 \%$ dos egressos estavam inseridos exclusivamente no setor público.

Entretanto, boa parte dos alunos vê o emprego público meramente como um complemento ou como um primeiro emprego, sugerindo uma visão onde o serviço público serve de escada para o mercado de trabalho individual e mercantilista. Dentro desta perspectiva, existe um claro esforço e direcionamento para a formação de profissionais de saúde bucal que atuem na lógica do Sistema Único de Saúde ${ }^{12}$.

Em um estudo realizado com estudantes do Curso de Graduação em Odontologia da
Universidade Federal do Pará, relatou-se que a maior parte dos deles vê o SUS como um espaço amplo de atendimento, de qualidade razoável, e direcionado à população ${ }^{18}$.

Em relação às adversidades, o presente estudo demonstrou que a maioria dos alunos espera encontrar dificuldades na profissão devido à saturação do mercado de trabalho e às condições financeiras da população. Esse quadro também foi observado na Universidade Federal da Paraíba, onde os alunos apresentaram expectativas semelhantes ${ }^{14}$. Uma possível saída seria a busca por uma melhor distribuição de profissionais, especialmente no interior, tanto para o excesso no mercado de trabalho quanto para à falta nas regiões com demandas não acolhidas.

A expectativa salarial dos estudantes mostra uma elevada pretensão, especialmente quando comparamos à renda do cirurgiãodentista, onde $49 \%$ dos profissionais recebem entre 1 a 3 mil reais mensais ${ }^{3}$, valor que apenas $8 \%$ dos participantes espera arrecadar, quadro observado também em outros estudos ${ }^{15}$. Tal quadro reforça a falta de conscientização sobre as questões financeiras da vida profissional. No estudo com egressos da Universidade Federal do Rio Grande do Norte, foi declarada uma renda que variava entre $R \$ 1.000,00$ e 5.400,00, embora maior parte recebesse acima de $\mathrm{R} \$ 3.400$, especialmente nos formados há mais de oito $\operatorname{anos}^{17}$.

O estudante de Odontologia da UFSC almeja continuar sua educação por meio da pósgraduação, manifestando essa vontade mesmo sem ter vivenciado o exercício da atividade profissional. $\mathrm{O}$ estudo dessa demanda, bem como das possíveis lacunas de conhecimentos e habilidades é necessário. Por outro lado, essa situação, que não é exclusiva da UFSC, pode indicar a busca por constante atualização e aperfeiçoamento $^{14}$. 
Ainda sobre este aspecto, observa-se que áreas clínicas e especializadas como a Prótese Dentária, a Dentística e a Implantodontia são as que despertam maior interesse para o estudo na pós-graduação entre os alunos, que são respectivamente a quinta, a sexta e a nona especialidade mais povoada de profissionais no Brasil $^{3}$. Em comparação, os estudantes da Universidade de São Paulo relataram alto interesse pela especialização, especialmente nas áreas de Cirurgia Bucomaxilofacial, Ortodontia e Prótese e Implantodontia ${ }^{15}$.

A procura pela Saúde Coletiva, entretanto, ainda é pequena, considerando que a maioria almeja um trabalho nesse setor, bem como os avanços do campo de trabalho para a Odontologia dentro do Sistema Único de Saúde e as possibilidades abertas por este no mercado de trabalho odontológico.

Em comparação a um estudo prévio realizado na mesma instituição, no ano de 2013, com estudantes de todos os semestres do Curso de Graduação em Odontologia, houve manutenção do perfil estudantil: predominou o sexo feminino, com faixa etária entre 18 e 24 anos, sendo maioria autodeclarada branca, solteira, e de classe A, B1 e B2. A maioria dos estudantes relatou que ainda morava com os pais ${ }^{13}$.

\section{CONCLUSÕES}

O último ano do Curso de Graduação em Odontologia da UFSC é majoritariamente composto por jovens, brancos, do sexo feminino, solteiros e sem filhos, provenientes de famílias com condições socioeconômicas favoráveis, sem necessidade de trabalhar para o próprio sustento, contando com o apoio familiar. Há uma clara polarização, mesmo observada a tendência para uma mudança nesse perfil para os próximos anos.

A maioria dos discentes veem no serviço público uma expectativa de atuação profissional, ainda que poucos apresentem uma perspectiva de trabalho exclusivo para a rede pública, tendo-a como um primeiro emprego ou uma "renda complementar" ao serviço privado. De forma geral, os alunos almejam uma média salarial maior do que a observada na realidade do estado de Santa Catarina, embora se preocupem com a evidente saturação de mercado, almejando a pósgraduação como forma de educação continuada.

\section{ABSTRACT \\ Profile and professional expectations among senior undergraduate Dental students from Federal University of Santa Catarina}

Brazilian dentistry experiences a time of constant changes. There is a highly competitive market, a growing Public Sector and new challenges for dentists. So, dental education needed to focus on a new academic profile, following the Brazilian National Curricular Guidelines. This study aimed at verifying the profile and professional expectations among fifth-year undergraduate dental students from Santa Catarina Federal University. A cross-sectional research was conducted with last year students, through a social-demographic and professional expectancies questionnaire. From 84 students, 76 participated in this study, resulting in a response rate of $90.48 \%$. The majority of dental students were women $(69.74 \%)$, white $(92.10 \%)$, single $(88,16 \%)$. The average age was 24 years. Most of the students came from private high school institutions $(64.47 \%)$, the monthly household income of 5-10 minimum wages $(39.47 \%)$ and did not work (84.21\%). Only $18.42 \%$ of students entered university through affirmative action. When regarding professional expectancies, $80.26 \%$ desires work in public sector, mainly because of stability $(42.10 \%)$ and as a supplementary income $(40.79 \%)$. Students mainly expect difficulty because of the highly competitive Market (69.74\%). All students reported a willingness to attend postgraduate studies, mainly in Dental Prosthesis (36.84\%), Aesthetic Dentistry (34.21\%) and Oral 
Implantology (32.90\%). This research enabled to verify a polarized profile among last year undergraduate dental students, who aim to work in public career. The main difficulty expected by students is the highly competitive Market.

Descriptors: Dental Education. Job Market. Dental Staff.

\section{REFERÊNCIAS}

1. Pinheiro CV, Menezes LMB, Aguiar ASW, Moura WVB, Almeida MEL, Pinheiro FMC. Inserção dos egressos do curso de Odontologia no mercado de trabalho. Rev Gaucha Odontol. 2011;277-83.

2. Freitas CHSM. Dilemas no exercício profissional da Odontologia: a autonomia em questão. Interface. 2007;11(21):25-38.

3. Morita MC, Haddad AE, Araujo ME. Perfil atual e tendências do cirurgião-dentista brasileiro. 1 ed. , Dental Press International. Maringá; 2010. 96 p.

4. Conselho Federal de Odontologia. Sistema de Cadastro: Geral no Brasil. 2016 [Acesso em 6 set. 2017]. Disponível em: www.cfo.org/br/ wpcontent/uploads/2011/06/TotalGeralBrasil.pdf

5. Matos PES, Tomita NE. A inserção da saúde bucal no Programa Saúde da Família: da universidade aos polos de capacitação. Cad Saude Pública. 2004;20(6):1538-44.

6. Sistema Único de Saúde - DataSUS. Quantidade de ocupações de Nível Superior segundo Região. 2016 [Acesso em 16 mar. 2016]. Disponível em: www.tabnet.datasus. gov.br

7. Haddad AE, Pierantoni CR, Ristoff D, Xavier I de M, Giolo J, Silva LB da. A trajetória dos cursos de graduação na saúde, 1991-2004. 2006. 533p.

8. Morita MC, Krieger L. Mudanças nos cursos de Odontologia e a interação com o SUS. Rev ABENO. 2004; 4(1):17-21.

9. Finkler M, Caetano JC, Ramos FRS. Ética e valores na formação profissional em saúde: um estudo de caso. Ciênc Saúde Colet. 2013;
18(10):3033-42.

10. Secco LG, Pereira MLT. Formadores em odontologia: profissionalização docente e desafios político-estruturais. Interface. 2004; 8(15):313-30.

11. Lazzarin HC, Nakama L, Cordoni Junior L. O papel do professor na percepção dos alunos de odontologia. Saúde Soc. 2007; 16(1): 90-101.

12. Carcereri DL, Amante CJ, Reibnitz MT, Mattevi GS, Silva GG, Padilha ACL, et al. Formação em Odontologia e interdisciplinaridade: o Pró-Saúde da UFSC. Rev ABENO. 2011;11(1):62-70.

13. Latreille AN, Sobrinho SM, Warmling AMF, Ribeiro DM, Amante CJ. Perfil socioeconômico dos graduandos em Odontologia da UFSC. Rev ABENO. 2015; 15(1):86-96.

14. Machado FC, Mariana D, Souto DA, Helena C, Freitas SM, Delano F, et al. Odontologia como escolha: perfil de graduandos e perspectiva para o futuro profissional. Rev ABENO. 2010;10(2):27-34.

15. Santos BNM, Gonzales PS, Carrer FCA, Araújo ME. Perfil e expectativas dos ingressantes da Faculdade de Odontologia da USP: uma visão integrada com as diretrizes curriculares nacionais e o sistema único de saúde. Rev ABENO. 2015; 15(1):28-37.

16. Unfer B, Rigodanzo L, Hahn D, Manfredini D, Rodrigues E, Cava CH. Expectativas dos acadêmicos de Odontologia quanto à formação e futura profissão. Saúde. 2004; 30(1-2):33-40.

17. Pinheiro IAG, Noro LRA. Egressos de Odontologia: o sonho da profissão liberal confrontado com a realidade. Rev ABENO. 2016 16(1):13-24.

18. Sales IT, Silva JM, Brandão AMM, Liliana SN, Brandão GAM. Percepção e estudantes de graduação em Odontologia sobre o Sistema de Saúde Brasileiro. Rev ABENO. 2016; 16(2): 69-76. 


\section{Correspondência para:}

Vinícius Spiger

e-mail: viniciusspiger@gmail.com

Rua Voluntários da Pátria, 2536 - Areias Brancas

97590-000 Rosário do Sul/RS 